\title{
Optical Characterization of ZnO Microsphere Produced by Laser Ablation in Air
}

\author{
Daisuke NAKAMURA, Norihiro TETSUYAMA, Tetsuya Shimogaki, Yuta SATO, Yasuaki MIZOKAMI, \\ Mitsuhiro HIGASHIHATA and Tatsuo OKADA \\ Graduate School of Information Science and Electrical Engineering, Kyushu University, \\ 744 Motooka, Nishi-ku, Fukuoka 819-0395, Japan \\ dnakamura@ees.kyushu-u.ac.jp
}

\begin{abstract}
We have succeeded in growing the $\mathrm{ZnO}$ microspheres by a simple laser ablation in the air, and we have obtained UV whispering-gallery-mode (WGM) lasing from the sphere under pulsed laser excitation. In this study, we observed resonances of WGM in visible region under $\mathrm{cw}$ laser pumping. In addition, possible refractive index of the $\mathrm{ZnO}$ microsphere was estimated from the WGM peaks. Furthermore, we obtained room-temperature electroluminescence from the $\mathrm{ZnO}$ microsphere/p-GaN heterojunction under forward bias.
\end{abstract}

DOI: $10.2961 / \mathrm{jlmn} .2015 .02 .0010$

Keywords: $\mathrm{ZnO}$, microsphere, whispering-gallery-mode, lasing, electroluminescence

\section{Introduction}

Zinc oxide $(\mathrm{ZnO})$ has a direct wide band-gap of 3.37 $\mathrm{eV}$ and a large exciton binding energy of $60 \mathrm{meV}$, which is much larger than the thermal energy at room temperature $(26 \mathrm{meV})$. Therefore, $\mathrm{ZnO}$ is one of the excellent candidate materials for ultraviolet (UV) emitting devices, such as UV light emitting diodes and UV lasers. In addition, $\mathrm{ZnO}$ nano/microstructures have attracted a great deal of attention as building blocks because of their high crystalline quality and unique structures. Those $\mathrm{ZnO}$ nano/microcrystals can be synthesized by several methods such as carbothermal CVD [1,2], hydrothermal method $[3,4]$, nanoparticles assisted pulsed laser deposition (NAPLD) [5-7], and so on. Interestingly, the $\mathrm{ZnO}$ nano/microcrystals can serve as good resonance cavities without additional mirrors due to the high refractive index of $\mathrm{ZnO}$. Light is confined in a single nano/microcrystal, and the oscillation route is formed within it. Lasing from $\mathrm{ZnO}$ nanowire [7-9], $\mathrm{ZnO}$ nanosheet [2], $\mathrm{ZnO}$ microdisk [10], $\mathrm{ZnO}$ ring [11], and $\mathrm{ZnO}$ nanonail [12] have been demonstrated under pulsed laser excitation. In our study, we have succeeded in synthesizing $\mathrm{ZnO}$ nano/microspheres by simple atmospheric laser ablation method, and demonstrated whispering-gallery-mode (WGM) lasing from the spheres $[13,14]$. In order to investigate the lasing properties of these nano/microcrystals, pulsed laser excitation is generally used because it induces population inversion due to the high peak power. Recently, we observed resonances of WGM in visible region from the $\mathrm{ZnO}$ microsphere under $\mathrm{cw}$ laser excitation, which is used for investigating the luminescence properties. In addition, room-temperature electroluminescence (EL) from the $\mathrm{ZnO}$ microsphere/p-GaN heterojunction under forward bias [15]. In this paper, we report the optical characteristics of optically- and electrically-pumped single $\mathrm{ZnO}$ microsphere. Furthermore, possible refractive index of the $\mathrm{ZnO}$ microsphere estimated from the WGM peaks are discussed.

\section{Experimental details}

\subsection{Synthesis of $\mathrm{ZnO}$ microsphere}

A $\mathrm{ZnO}$ microsphere synthesis method is laser ablation of a $\mathrm{ZnO}$ bulk target in the air, which is a quite simple laser process. The $\mathrm{ZnO}$ microspheres are synthesized by ablating the $\mathrm{ZnO}$ sintered target on which $\mathrm{Nd}$ :YAG laser beam $(\lambda=$ $1064 \mathrm{~nm}, 10 \mathrm{~Hz}$ ) was focused at a fluence of $5-40 \mathrm{~J} / \mathrm{cm}^{2}$. The atmospherically ablated $\mathrm{ZnO}$ droplets were collected on a proper substrate which was located at approximately 5 $\mathrm{mm}$ away from the focal point on the target, and then the spherical $\mathrm{ZnO}$ microcrystals were obtained. For a more detailed description of the $\mathrm{ZnO}$ microsphere synthesis, please refer to Refs. 13 and 14. The morphology of the microspheres were observed by scanning electron microscopy (SEM) and an optical microscope.

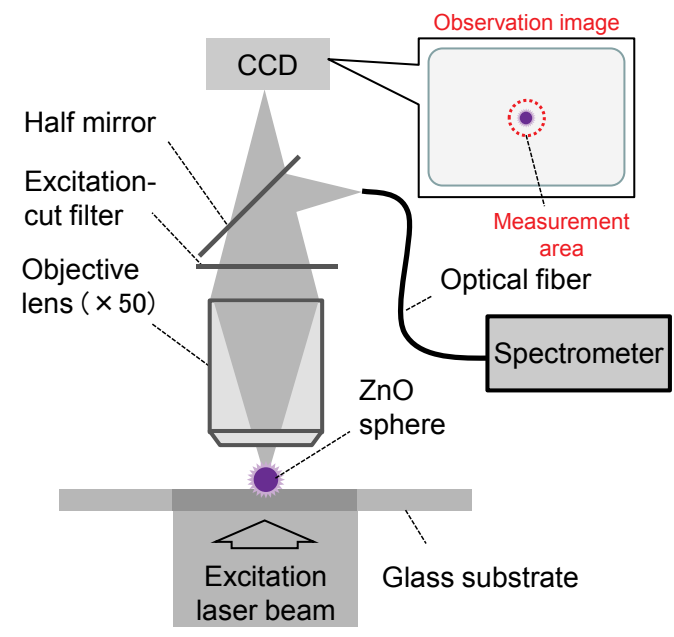

Fig. 1 Schematic of the microscopy system for measuring emission spectrum from a single $\mathrm{ZnO}$ microsphere. 


\subsection{Optical characterization of $\mathrm{ZnO}$ microsphere}

The emission from the $\mathrm{ZnO}$ microsphere was measured by a microscopic-spectroscopy system, as shown in Figure 1. The microspheres were excited by the third harmonics of a Q-switched Nd:YAG laser (355 nm, $5 \mathrm{~ns}$ ) or cw He-Cd laser. In this system, many microspheres dispersed on the substrate are excited at once. But, we can measure the emission from a single $\mathrm{ZnO}$ microsphere using an $\mathrm{x}-\mathrm{y}$ micro stage and a small observation area captured by an optical fiber.

\section{Results and discussion}

\subsection{Lasing characteristic of $\mathrm{ZnO}$ microsphere under pulsed laser excitation}

Fig. 2 shows the emission spectra from the $\mathrm{ZnO}$ microsphere excited by the Nd:YAG laser. Inset is the SEM image of the microsphere, which has a radius, $a$, of $1.6 \mu \mathrm{m}$. We have confirmed that the synthesized $\mathrm{ZnO}$ microspheres have wurtzite-structured $\mathrm{ZnO}$ crystal from the X-ray diffraction and micro-Raman measurement [13] Lasing spectra with modal structure from the $\mathrm{ZnO}$ microsphere were observed in UV region, which corresponds to the near band edge emission in $\mathrm{ZnO}$. These sharp peaks show a clear threshold behavior with the threshold power density of around $100 \mathrm{~kW} / \mathrm{cm}^{2}$, indicating that lasing took place within the microsphere. The mode spacing of the lasing spectra from the microspheres was $3.6 \mathrm{~nm}$, which corresponds to the WGM-theoretical mode spacing $\Delta \lambda$ expressed as the following equation [13]:

$$
\Delta \lambda=\frac{\lambda_{i}^{2}}{L}\left(n_{i}-\lambda_{i} \frac{d n_{i}}{d \lambda}+\frac{\lambda_{i}}{L}\right)^{-1}
$$

where $L$ is the cavity length, and $i$ is a modal number with an integer, $d n / d \lambda$ is the wavelength dispersion, and $n_{i}$ is the refractive index of the microsphere. The cavity length $L$ approaches to the circumference of the sphere if the modal number is large, resulting in $L \approx 2 \pi a$. The theoretical mode spacing of $3.64 \mathrm{~nm}$ was estimated around $\lambda_{m}=395 \mathrm{~nm}$, and the values of the refractive index and wavelength dispersion were $n_{i}=2.29$ and $d n_{i} / d \lambda=-0.0049 \mathrm{~nm}^{-1}$,

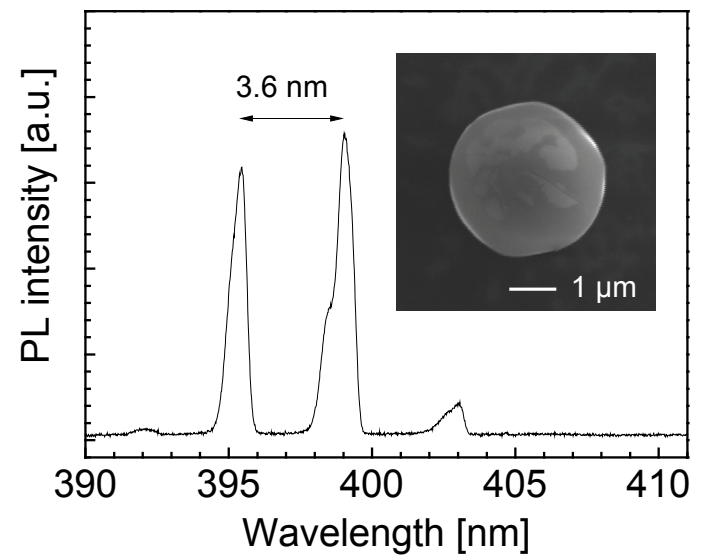

Fig. 2 Lasing spectra from the $\mathrm{ZnO}$ microsphere excited by the pulsed UV laser beam. Inset is the SEM image of the $\mathrm{ZnO}$ microsphere.

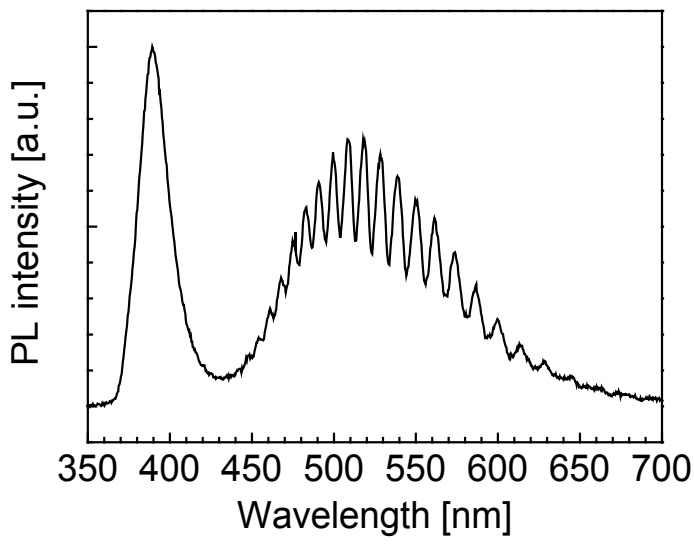

Fig. $3 \mathrm{PL}$ from the $\mathrm{ZnO}$ microsphere excited by cw He-Cd laser beam.

respectively [15], and it showed good agreement with the experimental result.

\subsection{Photoluminescence of $\mathrm{ZnO}$ microsphere under $\mathrm{cw}$ laser excitation}

Fig. 3 shows the photoluminescence (PL) from the $\mathrm{ZnO}$ microsphere with a diameter of $3.6 \mu \mathrm{m}$ under $\mathrm{cw} \mathrm{He}-\mathrm{Cd}$ laser $(325 \mathrm{~nm})$ excitation. A strong UV emission, which corresponds to the near-band-edge emission of $\mathrm{ZnO}$, and a broad visible emission with a modal peaks were observed. The visible emission was usually observed from $\mathrm{ZnO}$ under cw He-Cd laser excitation [2,16], and it is defect-related emission which is attributed to the deep-level oxygen vacancy $[17,18]$. It was confirmed that the modal peaks in visible region also correspond to WGMs from Eq. (1).

The WGMs in a spherical microcavity are characterized by a set of quantum numbers that describe the angular momentum mode number $i$ and radial mode number $l$. Assuming $a$ is much larger than $\lambda$, the relation between size parameter $x_{i}^{(l)}=2 \pi a / \lambda$ and $i, l$ can be expressed in powers of $v^{-1 / 3}[19,20]$ :

$$
n_{s} x_{i}^{(l)}=v-\zeta_{l}\left(\frac{v}{2}\right)^{\frac{1}{3}}+\sum_{k=0}^{k_{\max }} \frac{d_{k}\left(m, \zeta_{l}\right) m}{v^{1 / 3}\left(m^{2}-1\right)^{(k+1) / 2}}
$$

where $v=i+1 / 2$ and $m=n_{\mathrm{s}} / n_{\mathrm{e}}$ is the relative refractive index between the sphere and the surrounding medium. The coefficients $d_{\mathrm{k}}$ depend on the polarization (TM or TE) of the mode [20]. The $l$ th zero of the Airy function is denoted as $\zeta_{l}$.

Using Eq. (2), the refractive index of the $\mathrm{ZnO}$ microsphere was estimated because some papers reported that the dispersion of $\mathrm{ZnO}$ micro/nanocrystals was different from that of $\mathrm{ZnO}$ bulk or film $[10,12]$. In order to apply the $\mathrm{ZnO}$ microsphere to sensing devices [21], the absolute value of $n_{\mathrm{s}}$ is very important for its sensitivity and reliability. Fig. 4 shows the refractive indexes calculated using the experimental WGM peak wavelengths, where the first radial order $(l=0)$ and TE mode were estimated since they are more likely to be longer-lived than other WGMs [22]. In Fig. 4, different angular momentum mode number series were assigned, that is, $i=41-45$ at $\lambda=482.8 \mathrm{~nm}$. The dispersion curve with $i=43$ (@ $(\lambda=482.8 \mathrm{~nm})$ series is very close to that 


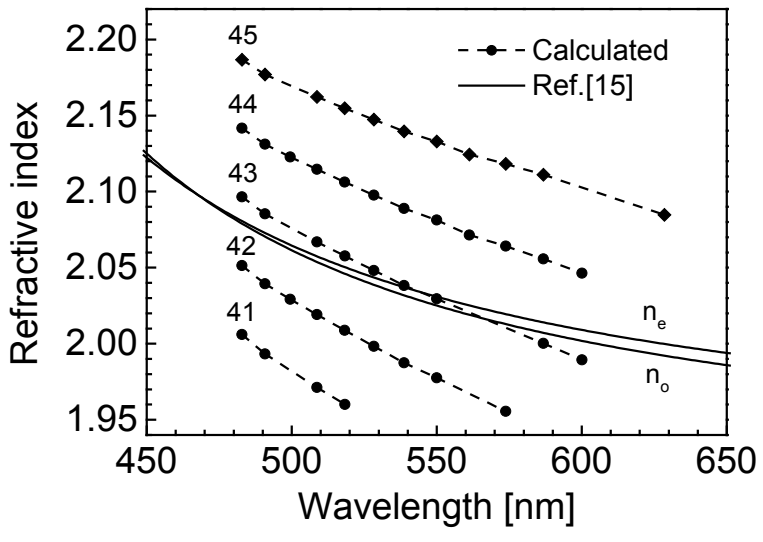

Fig. 4 Estimated refractive index of the $\mathrm{ZnO}$ microsphere.

of bulk $\mathrm{ZnO}$ [15], which probably shows the possible refractive index of the $\mathrm{ZnO}$ microsphere, because the dispersion of $\mathrm{ZnO}$ in visible region is small compared with that in UV region. The difference in the refractive index between the $\mathrm{ZnO}$ microcrystal and bulk $\mathrm{ZnO}$ was also reported $[12,22]$, though the reason remains unclear. The difference may be induced by the size of the $\mathrm{ZnO}$ crystal. Although a much more detail investigation is required, possible refractive index of the $\mathrm{ZnO}$ microsphere could be estimated from the WGM peaks.

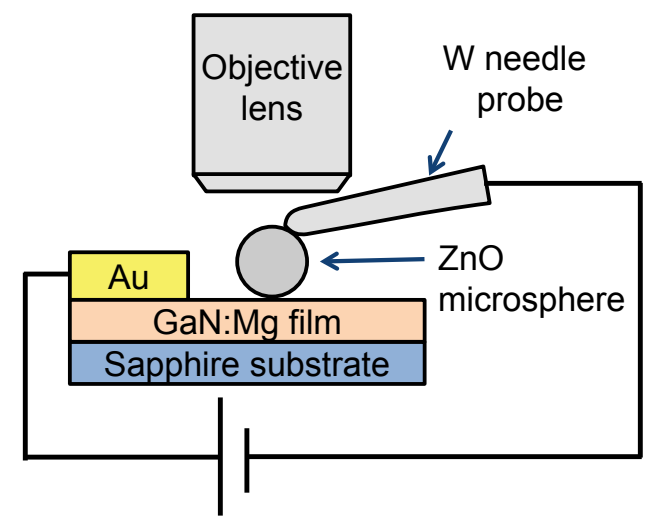

Fig. 5 Schematic of the $\mathrm{ZnO}$ microsphere/p-GaNfilm heterostructure for electrical excitation of the microsphere.

\subsection{Emission characteristic of $\mathrm{ZnO}$ microsphere under electrical excitation}

It is desirable to fabricate light emitting diodes and laser diodes based on $\mathrm{ZnO}$ microspheres to realize electrically pumped WGM emitter and lasing. Here, an optical property of the $\mathrm{ZnO}$ microsphere under electrical excitation was investigated. We fabricated the $\mathrm{ZnO}$ microsphere/p-GaN heterostructure, as shown in Fig. 5. In the experiment, the microsphere with a radius of $5 \mu \mathrm{m}$ was used. The $\mathrm{ZnO}$ microsphere and the $\mathrm{p}-\mathrm{GaN}$ film grown on the sapphire substrate formed a microsized junction on the interface. It was confirmed that Ohmic contacts were formed on the top $\mathrm{ZnO}$ buffer layer in contact with a $\mathrm{W}$ needle probe and the p-GaN film by depositing Au. EL emission of the $\mathrm{ZnO}$ microsphere/p-GaN heterostructure was observed under various forward biases, as shown in Fig. 6. Inset is the peak intensity at $400 \mathrm{~nm}$ as a function of the forward bias. The
near-UV emission peak at $400 \mathrm{~nm}$ was intensified with increasing the applied voltage up to $20 \mathrm{~V}$. The $I-V$ curves of the heterojunction clearly show the nonlinear increase of the current under forward bias. Rectifying characteristics with a threshold voltage of about $5 \mathrm{~V}$ was obtained, which is larger than that of nanowire-based heterojunction [23]. The heterojunction interface of the $\mathrm{ZnO}$ microsphere/p$\mathrm{GaN}$ film probably decreased the electron injection from the $\mathrm{ZnO}$ microsphere to the $\mathrm{p}-\mathrm{GaN}$ films due to poor contact, and reduced the electron-hole recombination when the forward bias is applied. Unfortunately, no WGM lasing from the $\mathrm{ZnO}$ microsphere was obtained though the EL emission was observed from the heterostructure. In this case, light generated at the heterojunction leaked to GaN film because the refractive index of $\mathrm{GaN}$ is larger than that of $\mathrm{ZnO}$ at $400 \mathrm{~nm}$. Other p-type material having a low refractive index will serve a high light confinement and lasing from the $\mathrm{ZnO}$ microsphere under electrical excitation.

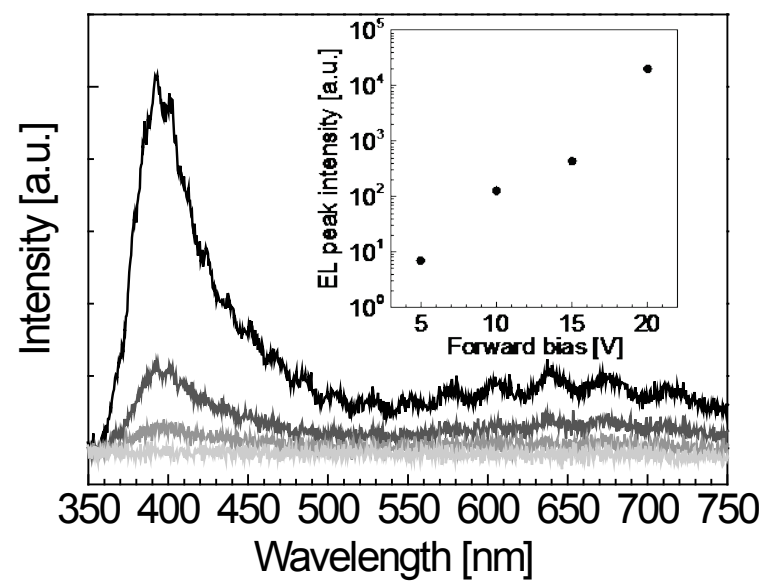

Fig. 6 EL spectra of the $\mathrm{ZnO}$ microsphere/p-GaNfilm heterostructure under forward bias. Inset is the peak intensities as a function of the forward bias.

\section{Conclusion}

We have achieved the $\mathrm{ZnO}$ microspheres by a simple atmospheric ablation method. The synthesized $\mathrm{ZnO}$ microspheres had wurtzite structure and completely spherical in shape. The UV WGM lasing of $\mathrm{ZnO}$ microspheres under pulsed laser excitation was obtained because of high lightconfinement property resulted from the spherical microcavity effect. In addition, WGM resonances in visible region was also observed from the $\mathrm{ZnO}$ microsphere pumped by a cw He-Cd laser. Experimental results were in good agreement with WGM theory. Using the WGM theory, possible refractive index of the $\mathrm{ZnO}$ microsphere was estimated from the experimental WGM peaks. Furthermore, room-temperature electroluminescence with peak wavelengths of $400 \mathrm{~nm}$ was observed from $\mathrm{ZnO}$ microspere/p$\mathrm{GaN}$ heterojunction under forward bias.

\section{Acknowledgments}

A part of this study has been financially supported by Grant-in-Aid for Scientific Research (B) from the Japan Society for the Promotion of Science (No. 25286071). 


\section{References}

[1] H. Wan and R. E. Ruda: J. Mater. Sci., 21, (2010) 1014.

[2] K. Okazaki, D. Nakamura, M. Higashihata, I. A. Palani and T. Okada: Opt. Exp., 19, (2011) 20389.

[3] L. Vayssieres: Adv. Materials, 15, (2003) 464.

[4] Y. Sun, N. G. Ndifor-Angwafor, D. J. Riley and M. N. R. Ashfold: Chem. Phys. Lett., 431, (2006) 352.

[5] M. Kawakami, A. B. Hartanto, Y. Nakata and T. Okada: Jpn. J. Appl. Phys., 42, (2003) L33.

[6] T. Okada, A. B. Hartanto and Y. Nakata: Appl. Phys. A, 79 (2004) 1417.

[7] Q. R. Guo, J. Nishimura, M. Matsumoto, D. Nakamura and T. Okada: Appl. Phys. A, 93, (2008) 843.

[8] A. M. Zimmler, F. Capasso, S. Muller and C. Ronning: Semicond. Sci. Technol., 25, (2010) 024001.

[9] K. L. Vugt, S. Rhle and D. Vanmaekelbergh: Nano Lett., 6(12), (2006) 2707.

[10]R. Chen, B. Ling, W. X. Sun and D. H. Sun: Adv. Mater., 23, (2011) 2199.

[11]J. P. Pauzauskie, J. D. Sirbuly and P. Yang: Phys. Rev. Lett., 96, (2006) 143903.

[12]J. Liu, S. Lee, Y. H. Ahn, J.-Y. Park, K. H. Koh and K. H. Park: Appl. Phys. Lett., 92, (2008) 263102.

[13]K. Okazaki, T. Shimogaki, K. Fusazaki, M. Higashihata, D. Nakamura, N. Koshizaki and T.
Okada: Appl. Phys. Lett., 101, (2012) 211105.

[14]D. Nakamura, T. Shimogaki, K. Okazaki, M. Higashihata, H. Ikenoue and T. Okada: J. Laser Micro/Nanoeng., 8, (2013) 296.

[15]H. Yoshikawa and S. Adachi: Jpn. J. Appl. Phys., 36, (1997) 6237

[16]S. C. Lyu, Y. Zhang, H. Ruh, H.-J. Lee, H.-W. Shim, E.-K. Suh, C. J. Lee: Chem. Phys. Lett., 363, (2002) 134

[17] S.B. Zhang, S.-H. Wei and A. Zunger: Phys. Rev. B, 63, (2001) 075205

[18] K. Vanheusden, C. H. Seager, W. L. Warren, D. R. Tallant and J. A. Voigt, Appl. Phys. Lett., 68, (1996) 403.

[19]S. Schiller and R. L. Byer: Opt. Lett., 16, (1991) 1138.

[20] S. Schiller: Appl. Opt., 32, (1993) 2181.

[21]R. S. Moirangthem and A. Erbe: Appl. Phys. Lett., 103, (2013) 051108.

[22]R. S. Moirangthem, P.-J. Cheng, P. C.-H. Chien, B. T. H. Ngo, S.-W. Chang, C.-H. Tien and Y.-C. Chang: Opt. Exp., 21 (2013) 3010.

[23]R. Guo, J. Nishimura, M. Matsumoto, M. Higashihata, D. Nakamura and T. Okada: Appl. Phys. B, 94, (2009) 33.

(Received: June 16, 2014, Accepted: February 25, 2015) 